\title{
Optimising prescription and titration of oxygen for adult inpatients using novel silicone wristbands: results of a pilot project at three centres
}

\author{
Authors: Sarah Forster, ${ }^{\mathrm{A}}$ Sue Smith, ${ }^{\mathrm{B}}$ Priya Daniel, ${ }^{\mathrm{C}}$ Amy Binnion, ${ }^{\mathrm{D}}$ Lucy Briggs, ${ }^{\mathrm{E}}$ Rachel Evans, ${ }^{\mathrm{F}}$ Kimuli Ryanna, ${ }^{\mathrm{F}}$ \\ Gerrit Woltmann, ${ }^{\mathrm{F}}$ Omar Bajammal, ${ }^{\mathrm{G}}$ David Hodgson, ${ }^{\mathrm{H}}$ Gauri Saini, ${ }^{\mathrm{I}}$ Jane Scullion, ${ }^{\mathrm{J}}$ Charlotte E Bolton ${ }^{\mathrm{K}}$ and \\ Gillian Lowrey ${ }^{\mathrm{L}}$
}

Oxygen is the most commonly used drug in the acute hospital setting. Oxygen can be lifesaving but there is increasing evidence that it can cause harm if it is not given correctly. Prescription of oxygen, according to target saturations, has been advocated since 2008 but compliance remains at low levels. This paper describes a novel approach to improve oxygen prescription and titration in three acute hospital trusts using a colour-coded silicone wristband. The project ran for 3 months and covered more than 2,000 emergency admissions to hospital. Data was collected for oxygen prescription and titration rates for 270 patients during the project period. The wristbands showed an improvement in prescription and titration of oxygen in two out of three sites. The results support a wider controlled study of colour-coded wristbands to improve oxygen safety in secondary care.

KEYWORDS: COPD, oxygen, patient safety, wristband

Authors: ${ }^{\text {A }}$ academic clinical fellow, Derby Teaching Hospitals NHS Foundation Trust, Derby, UK; 'B senior oxygen practitioner, Derby Teaching Hospitals NHS Foundation Trust, Derby, UK; ${ }^{C}$ respiratory registrar, Nottingham University Hospitals NHS Trust,

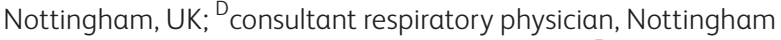
University Hospitals NHS Trust, Nottingham, UK; ${ }^{\text {Erespiratory }}$ specialist nurse, Nottingham University Hospitals NHS Trust, Nottingham, UK; ${ }^{\text {F }}$ consultant respiratory physician, University of Leicester Hospitals NHS Trust, Leicester, UK; ${ }^{\mathrm{G}}$ core medical trainee, University of Leicester Hospitals NHS Trust, Leicester, UK; ${ }^{\mathrm{H}}$ consultant respiratory physician, Sherwood Forest Hospitals NHS Foundation Trust, Nottingham, UK; ${ }^{\text {I }}$ consultant respiratory physician, Nottingham Respiratory Research Unit, School of Medicine, University of Nottingham, Nottingham, UK; ' ${ }^{\text {respiratory }}$ nurse consultant, University of Leicester Hospitals NHS Trust, Leicester, UK; ${ }^{K}$ clinical associate professor of respiratory medicine, Nottingham Respiratory Research Unit, School of Medicine, University of Nottingham, Nottingham, UK; ' consultant respiratory physician, Derby Teaching Hospitals NHS Foundation Trust, Derby, UK

\section{Introduction}

Oxygen is the most commonly used drug in the acute hospital setting. The 2013 UK oxygen audit organised by the British Thoracic Society ${ }^{1}$ showed that $13.8 \%$ of the 45,000 inpatients audited in acute hospital beds were on oxygen. However, only $52 \%$ of those receiving oxygen had a formal oxygen prescription with defined target oxygen saturations despite recommendations made by the National Patient Safety Agency in $2009^{2}$ that all patients receiving oxygen therapy should have a prescription in place.

Supplemental oxygen can save lives; however, inappropriate oxygen administration has been shown to have adverse outcomes. Hyperoxia is linked to increased mortality in stroke patients, ${ }^{3}$ intensive care and survivors of cardiac arrest. ${ }^{4}$ There is also evidence of increased long-term myocardial damage if uncontrolled oxygen therapy is administered during acute ST elevation myocardial infarction. ${ }^{5}$

One of the patient groups most at risk from excess oxygen therapy is those patients experiencing an acute exacerbation of chronic obstructive pulmonary disease (COPD). Controlled oxygen in the pre-hospital management of acute exacerbations of COPD is associated with reduced mortality and length of stay compared with patients who did not receive oxygen. ${ }^{6}$ The 2015 British Thoracic Society COPD care bundle project showed that receipt of the oxygen component of the bundle, including prescription, on hospital admission was associated with $80 \%$ lower inpatient mortality and reduced length of stay. ${ }^{7}$ However, the national COPD audit in $2014^{8}$ showed that only $55 \%$ of patients admitted with acute exacerbation of COPD had oxygen prescribed, demonstrating the need for improvement in this area. In 2013, a quality improvement project was performed on the acute respiratory wards at the Royal Derby Hospital to assess whether the introduction of coloured silicone wristbands to indicate the patient's target oxygen saturations, could improve compliance with British Thoracic Society oxygen guidelines. ${ }^{9}$ This project took place from August to October 2013 with education provided to the staff followed by the introduction of the wristbands. The project demonstrated improved prescription of oxygen (with target saturations) from $85 \%$ in 2012 to $95 \%$ in 2013. There was also an improvement in patients having oxygen saturations within the prescribed target 
range from $78 \%$ in 2012 to $85 \%$ in 2013. Contemporaneous staff surveys demonstrated a $50 \%$ increase in awareness of the importance of careful oxygen titration and patient feedback demonstrated the acceptability and comfort of the wristbands, and that patients were reassured by them.

For such an intervention to be relevant on a wider scale in the UK, it was imperative to assess its effectiveness in different acute hospital settings. The project was therefore assessed in three acute hospitals across the East Midlands, all with different working practices. By improving the prescription of target saturations and titration of oxygen within the acute hospital setting, the aim was to reduce incidents of oxygen toxicity and thereby have a positive impact on morbidity, mortality and length of stay, all of which have potential cost saving implications.

\section{Methods}

There were three acute hospital settings involved in the project:

1 Respiratory Medicine Department, Nottingham City Hospital Campus, Nottingham University Hospitals NHS Trust - receives secondary and tertiary referrals, usually through an assessment unit, but also direct transfers crosssite to respiratory wards, and part of the two-site Nottingham University Hospitals NHS Trust.

2 Glenfield Hospital, Leicester - a tertiary centre and part of the two-site University Hospitals of Leicester NHS Trust.

3 Respiratory wards at King's Mill Hospital, Mansfield primary site of the Sherwood Forest NHS Foundation Trust.

The quality improvement project ran for 16 weeks, supported by the East Midlands Respiratory Programme and funded by a 2014 East Midlands Academic Health Science Network innovation award. Ethics approval and informal consent was not required as this project was run as a quality improvement project. However, a risk assessment was carried out at each site and the project approved by the relevant local governance committees. A key governance issue raised was potential overlap of the colours proposed with existing bands at each of the hospitals involved. Following discussions with local governance committees, the colour choice of the wristbands was agreed across the three sites (Fig 1). Methods for data collection on adverse events were also agreed.

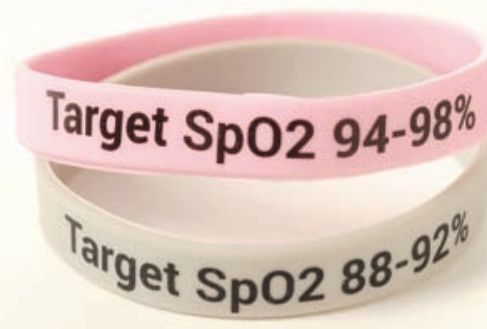

Fig 1. Colour-coded silicone wristbands. Pink = target oxygen saturation of $94-98 \%$; grey = target oxygen saturation of $88-92 \%$.
Training was provided to all staff across all three trusts during the month before the project commenced in the form of a centrally designed presentation outlining the project and the reasons for carrying it out, and the roles of the ward staff while the project was running (Fig 2). Reminder posters were placed on trolleys and in other areas where the project was running. An oxygen champion was identified to support the project in each area.

The project ran from 13 April to 31 July 2015. All patients admitted within the specified areas participating in this project within these trusts had oxygen prescribed according to target saturations as per current British Thoracic Society guidelines, and were given the appropriate coloured wristband. Leicester Glenfield and Nottingham City Hospital campus used the wristbands from the point of admission on the admission units within the trust site; King's Mill Hospital applied the wristbands for all admissions transferred to the respiratory wards. The doctor was responsible for prescribing oxygen and target saturations on admission; the nurse then applied the appropriate wristband at the first drug round or during triage (Glenfield) (Fig 3).

Over the 16 weeks, three assessments were conducted, each over 4 days:

1 start of project, before training (March 2015)

2 midpoint (mid-June 2015)

3 final (end of July 2015).

Assessment was based on the British Thoracic Society oxygen audit. ${ }^{1}$ Outcome measures were judged to have been met based on written documentation in medical and nursing notes and electronic observations (Box 1).

In addition, COPD mortality and length of stay from monthly hospital episode statistics (HES) data was collected for COPD inpatients. Where available (Nottingham and King's Mill Hospital) the percentage of arterial blood gases taken where $\mathrm{pO}_{2}>13 \mathrm{kPa}$ was recorded weekly as an indicator of over oxygenation and collected in an automated manner from the blood gas machines in two out of three hospital sites. Staff surveys were designed by the project team and were conducted at the end of the 16 weeks. At discharge, patients were asked to give feedback according to a structured short feedback questionnaire.

\section{Results}

In excess of 2,000 emergency admissions passed though the project areas during the 16-week period. All sites carried out the assessments before, at the midpoint and the end of the trial period within a 4-day period of each other. Oxygen prescription and titration data was collected for 270 patients across the three sites. There was an improvement in the percentage of patients with an oxygen prescription at Glenfield (98\% at the end versus $64 \%$ before) and King's Mill ( $94 \%$ at the end versus $76 \%$ before). Conversely, Nottingham did not show an improvement in oxygen prescription $(69 \%$ at the end versus $74 \%$ before). Of those who had an oxygen prescription in place, there was an improvement in the proportion of patients within the target range at Glenfield ( $98 \%$ at end verses $61 \%$ before) and King's Mill (94\% at end versus 52\% before). There was no improvement in patients within the target range at Nottingham City Hospital (22\% at end versus $41 \%$ before) (Table 1 ). 


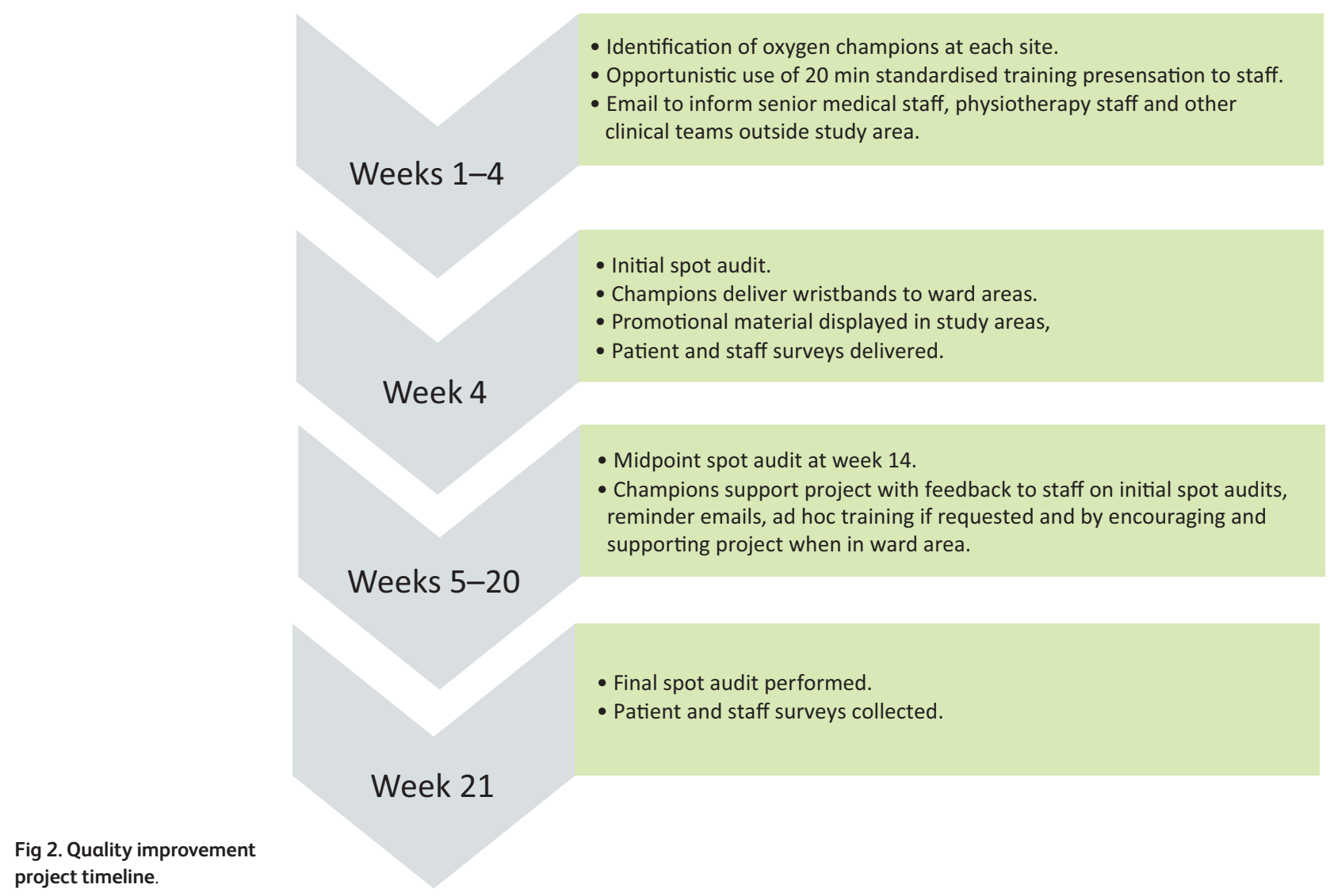

Arterial blood gas results were only available from two sites (Nottingham City Hospital and King's Mill Hospital) and there was no reduction in $\mathrm{pO}_{2}$ seen.

The patient survey was completed by 192 patients across the three sites with a largely positive response to the intervention. The staff survey was completed by 186 members of staff across healthcare professional grades and roles. Of the members of staff who completed the survey, $86 \%$ felt the wristbands added

\section{Box 1. Outcome measures}

Number of patients receiving oxygen (denominator).

Number of patients with oxygen prescription (numerator).

Number of patients within the target saturations (numerator).

Percentage of arterial blood gas sampling where $\mathrm{pO}_{2}>13 \mathrm{kPa}$.

Mortality and length of stay for COPD (HES data).

Additional subjective elements:

$>$ Patient survey assessing understanding, improvement in knowledge, comfort and reassurance.

> Staff survey assessing the response of each member of the ward team as to whether they felt the intervention helped to manage patient oxygen usage.

COPD = chronic obstructive pulmonary disease; HES = hospital episode statistics. at least some benefit and $46 \%$ felt it helped significantly. HES data for length of stay and crude mortality was collected for 1,077 coded admissions with acute exacerbations of COPD. The data was for all admissions and not confined to those within the project areas. There was no significant change in either mortality or length of stay during the 16 -week project period.

\section{Discussion}

The results of this quality improvement project are in broad agreement with the pilot project, and show that the use of colour-coded oxygen saturation wristbands is effective at improving the safe prescription of oxygen to inpatients. The project resulted in an increase in the proportion of patients with oxygen prescribed and the time the patient spent in the target saturation range.

The wristbands used have several benefits over other methods of documentation in that they are clearly visible to all, likely to remain with the patients at all times, easily understandable by all members of the team and are comfortable for the patients to wear - an important issue for compliance. Many patients continued to wear their wristbands post discharge potentially conferring benefit on subsequent admissions and in the prehospital setting. The wristbands are also low cost (13p per band).

Tuner et al showed correct use of oxygen was associated with a reduced length of stay in those admitted with acute exacerbations of COPD. ${ }^{7}$ Admissions for acute exacerbations 


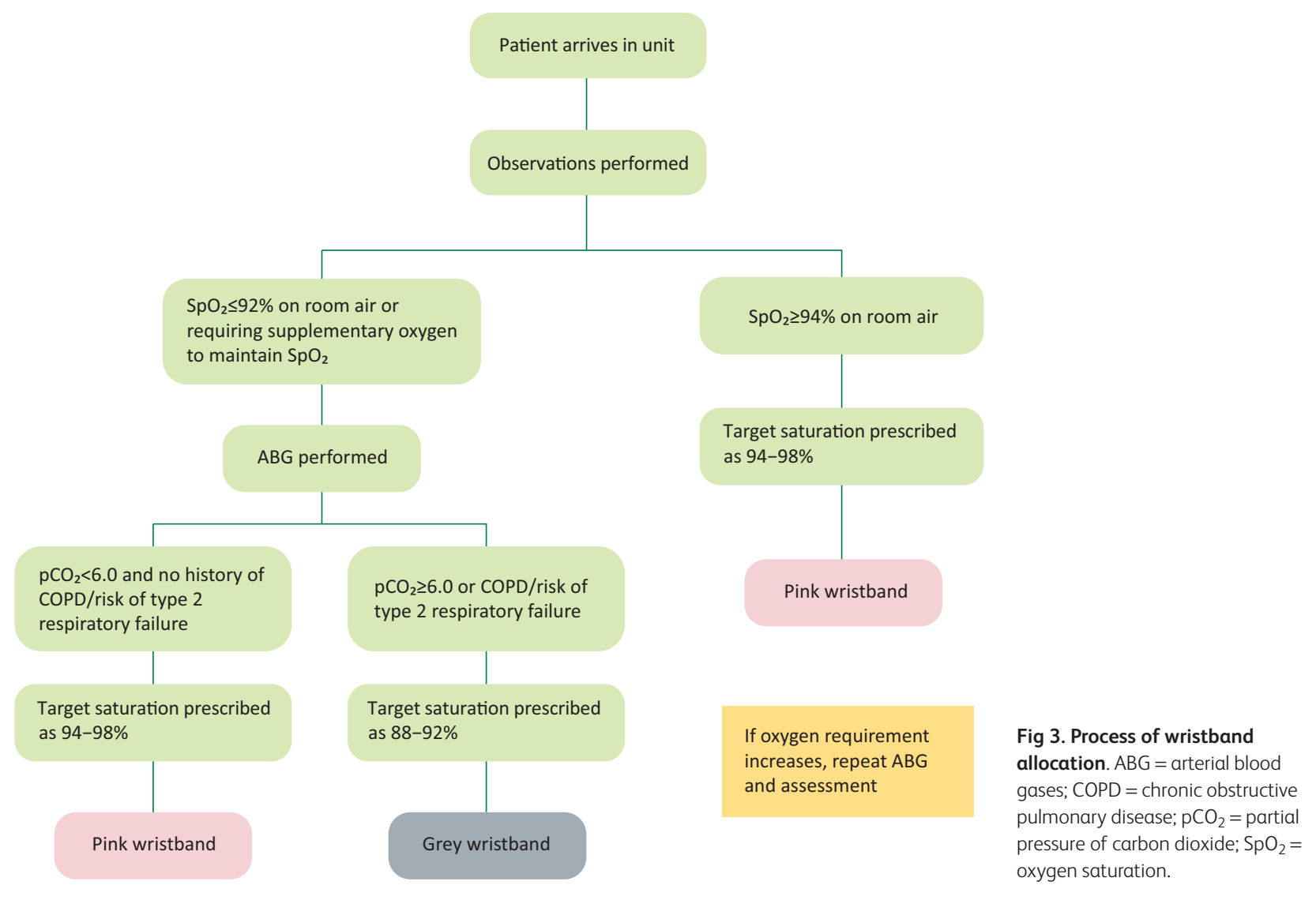

of COPD continue to rise and place a significant burden on healthcare resources. ${ }^{8}$ Improving oxygen use could reduce the costs associated with patient admission.

This project highlights that site-specific implementation of wristbands is important for improving outcomes and success. The project was implemented with strong championing from middle grade and senior medical staff at all three sites. While all sites showed improvements in patient and staff understanding, only two out of the three sites showed improvements in oxygen prescription and titration rates; Glenfield Hospital and King's Mill Hospital. At Glenfield Hospital all patients, including intersite transfers, pass though the cardiorespiratory admissions unit. At King's Mill Hospital, the project ran on the respiratory wards only.
Nottingham City Hospital campus ran the project on all five of its respiratory wards, including the respiratory admissions ward. However, at this pilot stage, the project was not extended to the general medical receiving units at the Queen's Medical Centre campus. In contrast to Leicester, intersite transfers of respiratory patients from Queen's Medical Centre campus do not pass through the respiratory admissions ward at Nottingham City Hospital campus but transfer directly to the respiratory base wards. Interestingly, a differential effect was seen whereby $100 \%$ compliance with oxygen prescribing was seen on the respiratory admissions ward, but this compliance was substantially lower on the respiratory base wards (data not shown) suggesting that the reduction in compliance was due to

\section{Table 1. Audit of oxygen prescription across the three assessment periods.}

\begin{tabular}{|c|c|c|c|c|c|c|c|c|c|}
\hline & \multicolumn{3}{|c|}{ Nottingham } & \multicolumn{3}{|c|}{ Glenfield } & \multicolumn{3}{|c|}{ King's Mill } \\
\hline & 1st & 2nd & $3 r d$ & 1st & 2nd & 3rd & 1st & 2nd & $3 r d$ \\
\hline Patients receiving oxygen, $n=270$ & 35 & 25 & 32 & 36 & 44 & 40 & 25 & 16 & 17 \\
\hline Patients with oxygen prescription, $\mathrm{n}=220$ (\%) & $26(74)$ & $18(72)$ & $22(69)$ & $23(64)$ & $43(98)$ & $39(98)$ & $19(76)$ & $14(88)$ & $16(94)$ \\
\hline Patients with prescription of $94-98 \%$ & 9 & 6 & 5 & 10 & 21 & 19 & 8 & 6 & 4 \\
\hline Patients with prescription of $88-92 \%$ & 15 & 10 & 14 & 9 & 21 & 20 & 9 & 5 & 8 \\
\hline Patients with other prescription & & & & & & & 0 & 3 & 4 \\
\hline $\begin{array}{l}\text { Patients in prescribed target saturation range } \\
n=176(\%)\end{array}$ & $14(40)$ & $10(40)$ & $7(22)$ & $22(61)$ & $43(98)$ & $39(98)$ & $13(52)$ & $12(75)$ & $16(94)$ \\
\hline
\end{tabular}


the receipt of patients from medical receiving wards that were not included in the pilot project. This suggests that inclusion of all medical receiving units may have resulted in a more positive outcome and, importantly, that inclusion of those units would be required for a wider roll out to be successful.

It is important to acknowledge other limitations of the project. The project involved training for staff, developing oxygen champions and promotion alongside the wristbands. It is not possible to say how much of the improvement seen was due to the educational package and champions and how much was due to the wristbands themselves. It would be important to consider this when designing future studies, developing control arms to examine this.

Not all of the information requested was available from all three of the centres. Only two of the three sites were able to provide arterial blood gas data for logistical reasons. Secondly, the blood gas values do not specify at what stage in a patient's admission these were taken and therefore their significance in relation to this project is limited; for example, an at the door test would reflect inappropriate oxygen delivery prior to the wristband being applied and would therefore not be affected by the intervention. If some of the high oxygen levels are indeed through pre-hospital oxygen delivery, it would be interesting to see the longer term impact of the wristbands on prehospital oxygen delivery, since some patients reported that they intended to continue wearing their wristbands after discharge. However, it is important to note that a significant number of patients had $\mathrm{pO}_{2}>13 \mathrm{kPa}$ on two sites where data were available, suggesting some degree of oxygen toxicity within the acute hospital setting.

Mortality and length of stay data did not show any changes during the implementation of the wristbands at any of the three sites. It is important to note the project's primary aim was to improve process measures. Outcome measures were collected to reassure us that there were no detrimental effects to this group of patients (acute exacerbation of COPD) where inappropriate oxygen treatment carries a high risk of harm. ${ }^{6}$

It is also necessary to consider the comments of staff involved in implementing the trial. For example, oxygen wristbands are not a substitute for prescription of oxygen as a drug on the medications chart and clear documentation of target saturations. However, they do provide a continually visual reminder to all members of the multidisciplinary team of the need for appropriate oxygen delivery for each individual patient.

This project concentrated on respiratory wards and acute cardiorespiratory admission units. The project showed improvement in prescription during a defined project period. There is a bigger challenge to maintain the change outside of a project period and outside of the respiratory specialty areas. The initial site to implement the wristbands, Derby Teaching Hospitals NHS Foundation Trust, has been able to maintain prescription levels of $95 \%$, with more than $90 \%$ within the target range by continuing to use the wristbands on the respiratory wards. In these areas, all patients have both a target saturation prescribed and a wristband, regardless of whether they are receiving oxygen. However, roll out of the wristbands alone, without the 'prescription for all' policy, in the emergency department and medical assessment areas in this trust have not been able to maintain high prescription and titration rates. Using a combined approach of prescription and wristband to all patients in acute inpatient areas may provide a solution to improving the safety of oxygen in secondary acute care but this needs to be examined outside respiratory wards and in areas where oxygen use is less prevalent and the perceived harm from oxygen toxicity is less recognised.

\section{Conclusions}

The project shows that coupled with active oxygen champions, oxygen saturation wristbands can improve prescription and titration of oxygen in a variety of acute hospital settings. We would recommend further roll out of this project to other acute hospitals in order to improve oxygen safety in acute care settings.

\section{Conflicts of interest}

The authors have no conflicts of interest to declare.

\section{Acknowledgements}

East Midlands Respiratory Programme helped to coordinate the logistics of the study and the money from the East Midlands Academic Health Science Network innovation prize funded the wristbands for all three sites.

\section{Funding}

Funding was provided by an innovation award from East Midlands Academic Health Science Network in 2014.

\section{References}

1 O’Driscoll BR. Emergency oxygen audit 2013. London: British Thoracic Society, 2013. Available at www.brit-thoracic.org.uk/ document-library/audit-and-quality-improvement/audit-reports/ bts-emergency-oxygen-audit-report-2013/ [Accessed 4 May 2016].

2 National Patient Safety Agency. Oxygen safety in hospitals. Rapid response report NPSA/2009/RRR006. London: NPSA, 2009.

3 Rønning OM, Guldvog B. Should stroke victims routinely receive supplemental oxygen: a quasi-randomised controlled trial. Stroke 1999;30:2033-7.

4 Kilgannon JH, Jones AE, Shapiro NI et al. Association between arterial hyperoxia following resuscitation from cardiac arrest and inhospital mortality. JAMA 2010;303:2165-71.

5 Stub D, Smith K, Bernard S et al. Air versus oxygen in ST-segmentelevation myocardial infarction. Circulation 2015;131:2143-50.

6 Austin MA, Wills KE, Blizzard L et al. Effect of high flow oxygen on mortality in chronic obstructive pulmonary disease in prehospital setting: randomised controlled trial. BMJ 2010;341:c5462.

7 Turner AM, Lim WS, Rodrigo C, Welham Calvert JM. A care-bundle approach to improving standards of care in AECOPD admissions: results of a national project. Thorax 2015; 70:992-4.

8 National COPD Audit Programme. COPD: Who cares matters. London: Royal College of Physicians, 2015.

9 O'Driscoll BR, Howard LS, Davidson AG. Emergency use of oxygen in adult patients guideline. BMJ 2008;63(Suppl 6):vi1-68.

Address for correspondence: Dr G Lowrey, Department of Respiratory Medicine, Derby Teaching Hospitals NHS Foundation Trust, Uttoxeter Road, Derby DE22 3NE, UK. Email: gillian.lowrey1@nhs.net 